\title{
Dysregulation of ion homeostasis by antifungal agents
}

\author{
Yongqiang Zhang, Sabina Muend and Rajini Rao* \\ Department of Physiology, The Johns Hopkins University School of Medicine, Baltimore, MD, USA
}

\section{Edited by:}

Bruce C. Campbell, Western Regional Research Centre, USA

\section{Reviewed by:}

Bruce C. Campbell, Western Regional

Research Centre, USA

Jong H. Kim, United States

Department of Agriculture-Agricultural

Research Service, USA

\section{*Correspondence:}

Rajini Rao, Department of Physiology, The Johns Hopkins University School of Medicine, 725 North Wolfe Street, Baltimore, MD 21205, USA.

e-mail: rrao@jhmi.edu
Ion-signaling and transduction networks are central to fungal development and virulence because they regulate gene expression, filamentation, host association, and invasion, pathogen stress response and survival. Dysregulation of ion homeostasis rapidly mediates cell death, forming the mechanistic basis by which a growing number of amphipathic but structurally unrelated compounds elicit antifungal activity. Included in this group is carvacrol, a terpenoid phenol that is a prominent component of oregano and other plant essential oils. Carvacrol triggers an early dose-dependent $\mathrm{Ca}^{2+}$ burst and long lasting $\mathrm{pH}$ changes in the model yeast Saccharomyces cerevisiae. The distinct phases of ionic transients and a robust transcriptional response that overlaps with $\mathrm{Ca}^{2+}$ stress and nutrient starvation point to specific signaling events elicited by plant terpenoid phenols, rather than a non-specific lesion of the membrane, as was previously considered. We discuss the potential use of plant essential oils and other agents that disrupt ion-signaling pathways as chemosensitizers to augment conventional antifungal therapy, and to convert fungistatic drugs with strong safety profiles into fungicides.

Keywords: Candida albicans, plant essential oils, terpenoid phenol, carvacrol, amiodarone, calcium burst, pH, vacuole

\section{URGENCY IN THE NEED FOR ANTIFUNGAL INTERVENTION}

Invasive fungal infections are on the rise worldwide, in parallel with increasing populations of immune-compromised individuals, common use of catheters and medical implants, and overprescription of antifungals and steroid therapy (Gudlaugsson et al., 2003; Francois et al., 2005; Ashley et al., 2006; Pappas et al., 2009; Morace and Borghi, 2010). As eukaryotes, fungi share essential metabolic pathways and core cellular machineries with humans so that pathogen-selective targets are scarce. At present, antifungal therapy is limited to a few classes of chemically distinct drugs that are handicapped by emergence of resistant phenotypes, concomitant toxicity, and substantial inter-patient variations in serum drug levels and efficacy (Francois et al., 2005). This highlights the pressing need for alternative treatments by exploring unconventional classes of antifungal agents and defining novel drug targets.

\section{ION-SIGNALING PATHWAYS IN FUNGAL PATHOPHYSIOLOGY AND VIRULENCE}

One cellular pathway that has received less attention than it deserves is that of ion homeostasis. Ionic signal transduction networks are central to the regulation of fungal development and pathogenicity (Davis, 2009; Lew, 2011). Prominent among these are $\mathrm{Ca}^{2+}$ cascades that originate from plasma membrane channels and receptors and link to $\mathrm{Ca}^{2+}$ sensors and transcription factors that regulate gene expression, filamentation, host association, and invasion (Kraus and Heitman, 2003; Blankenship and Heitman, 2005; Wang et al., 2011). Key cytoplasmic players include the $\mathrm{Ca}^{2+}$ binding protein calmodulin, Ser/Thr phosphatase calcineurin, and the transcription factor Crz1. Membrane-embedded $\mathrm{Ca}^{2+}$ influx channels and efflux pumps make excellent targets for drugs that partition into the bilayer. Similarly, $\mathrm{H}^{+}$homeostatic pathways are also key regulators in pathogenesis. We recently identified the Vtype $\mathrm{H}^{+}$-ATPase as a major downstream target of widely used azole drugs (Zhang et al., 2010). Fluxes of $\mathrm{K}^{+}$and $\mathrm{Na}^{+}$modulate membrane potentials and $\mathrm{pH}$ gradients that profoundly affect downstream signaling pathways, metabolic activity, and vesicle trafficking (Soong et al., 2000; Brett et al., 2005). Compartmentspecific ionic signaling separates critical events in mitochondria, vacuoles, and secretory pathway from the cytoplasm. Because dysregulation of ion homeostasis rapidly mediates cell death, we have been exploring how ion-signaling pathways may serve as targets for antifungal drugs.

\section{PLANT ESSENTIAL OILS, PAST AND FUTURE}

Herbal remedies have been recognized since ancient times, but in the last decade, increasingly sophisticated analytical tools have been brought to bear on their mechanism of action. Plant essential oils demonstrate microbicidal activity against a wide range of fungal and bacterial pathogens, including Pseudomonas aeruginosa, Candida albicans, and Staphylococcus aureus (Ben Arfa et al., 2006; Pozzatti et al., 2008; De Martino et al., 2009; Pinto et al., 2009). A survey of the literature revealed that essential oils derived from the genus Oreganum were among the most effective of herbal extracts tested, with minimum inhibitory concentration (MIC) of 500 parts per million against C. albicans (Tampieri et al., 2005). A major component of plant essential oils, including Oregano extract is the terpenoid phenol class of compounds that include carvacrol, eugenol, and thymol. Each of these has been demonstrated to exhibit potent antifungal activity on its own, not only against planktonic fungi but also biofilms and drug resistant mutants (Dalleau et al., 2008). 


\section{PHYSICOCHEMICAL PROPERTIES OF ESSENTIAL OIL COMPOUNDS CORRELATE WITH ANTIFUNGAL EFFICACY}

Many membrane-active antifungal agents are cationic amphiphilic compounds that share common physicochemical properties of a hydrophobic ring structure and a hydrophilic side chain with a charged cationic group, such as an amine. The hydrophobic portion ensures a high partition coefficient into lipid membranes. Whereas, their hydrophilic and charged moieties retain them in the bilayer where they induce membrane changes that have important consequences for ion homeostasis and signaling. This amphiphilic property is illustrated by the chemical structures of terpenoid phenols (Figure 1). Another such compound is the antiarrhythmic agent, amiodarone that also exhibits antimycotic activity through similar cellular effects on ion homeostasis (Gupta et al., 2003; Muend and Rao, 2008).

An evaluation of the relative antifungal efficacy of these terpenoid phenols and their related compounds revealed an ordering of carvacrol $\geq$ thymol $\gg$ eugenol $\gg \gamma$-terpinene, with vanillin and guaiacol being ineffective. Similar results were reported in other studies (Voda et al., 2004). Carvacrol exhibited a MIC of $0.008 \%$ (or $79.8 \mu \mathrm{g} / \mathrm{ml}$ ) against Saccharomyces cerevisiae, which was 1,500 times more effective than Oregano oil. The lowered efficacy of eugenol appears to be linked to the methylation of its hydroxyl group. Similarly, hydroxymethyl derivatives of carvacrol, thymol, and eugenol had significantly lower antifungal activity relative to the parent compounds (Mastelic et al., 2008). However, hydrophobicity alone does not ensure toxicity since $p$-cymene, a precursor of carvacrol, has a higher partition coefficient for lipid membranes than carvacrol but is non-toxic. The presence of the hydroxyl group is critical for toxicity, as seen by lack of microbicidal effects of $p$-cymene and carvacrol methylesters (Bagshaw, 1983; Ultee et al., 2002). This suggests that antifungal activity depends on the structure and make-up of the terpenoid phenols, specifically the presence of a free hydroxyl group and an aromatic ring.

\section{BILAYER POLARIZATION AND EFFECTS ON MEMBRANE POTENTIAL}

A first step in their mechanism of action is the preferential partitioning of the compound into the lipid membrane: thus, carvacrol has a $\log P$ value of 3.26 for partition into phosphatidylethanolamine membranes relative to the buffer (Ultee et al., 2002). It has been proposed that the delocalized electron system in carvacrol facilitates the dissociation of $\mathrm{H}^{+}$from the $\mathrm{OH}$ group. This, in turn, would allow carvacrol to shuttle $\mathrm{H}^{+}$and monovalent cations such as $\mathrm{K}^{+}$across membranes, dissipating pH and $\mathrm{K}^{+}$gradients across cell membranes (Ultee et al., 2002). Consistent with this mechanism, carvacrol was also shown to depolarize bacterial cell membranes and decrease accumulation of the fluorescent dye 5(6)-carboxyfluorescein diacetate, suggestive of an increase in membrane permeability (Xu et al., 2008). Using the potential sensitive fluorescence probe diS- $\mathrm{C}_{3}(3)$ to monitor membrane potential changes in exponentially growing yeast cells, we showed that the amphiphilic drug amiodarone caused a dosedependent hyperpolarization of the plasma membrane, consistent with increased activity of the plasma membrane $\mathrm{H}^{+}$-ATPase and outwardly rectifying $\mathrm{K}^{+}$channels (Maresova et al., 2009). Above a drug threshold of $4 \mu \mathrm{M}$, however, amiodarone depolarized the membrane potential and this correlated with loss in viability. Loss of function mutations in PMA1 (plasma membrane $\mathrm{H}^{+}$-ATPase) and deletion of the TOK1 (outward-rectifier potassium channel of the plasma membrane) gene reduced the initial hyperpolarization and also protected against amiodarone mediated cell death. Thus membrane potential increases were linked to and essential for drug toxicity, confirmed by the depolarizing and protective effects of salts, such as $\mathrm{KCl}$ (Maresova et al., 2009).

Cationic amphiphiles also alter membrane fluidity, which in turn could tune the activity of membrane proteins, including ion channels and transporters. Amiodarone was found to have an ordering effect on the lipid bilayer, as seen by changes in fluorescence polarization of 1,6-diphenyl-1,3,5-hexatriene (DPH; Antunes-Madeira et al., 1995). Braga and Ricci (2011) used atomic

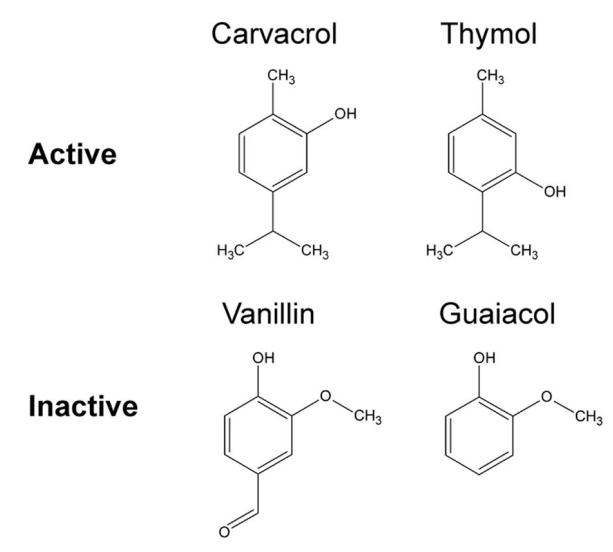

FIGURE 1 | Structures of terpenoid phenols found in plant essential oils, with closely related precursors. A subset of these compounds elicit $\mathrm{Ca}^{2+}$ and $\mathrm{pH}$ transients and are active as antifungals. Compounds with $\mathrm{MIC}$ greater
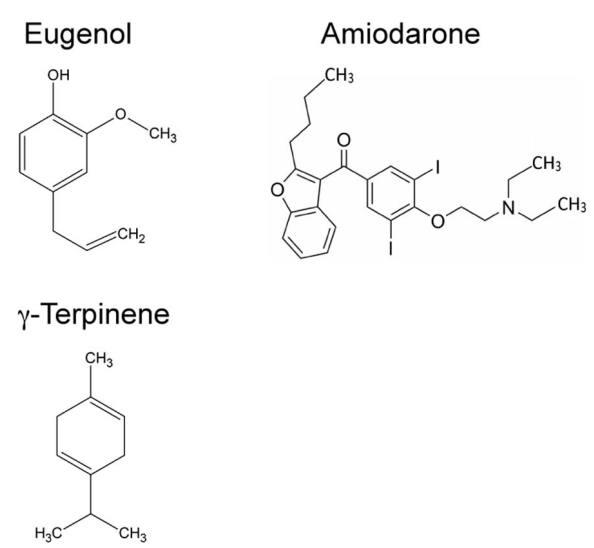

than $0.5 \mathrm{mg} / \mathrm{ml}$ are grouped as inactive. Amiodarone, a cationic amphipathic drug used to treat cardiac arrhythmia that moonlights as an antifungal agent, also elicits ion dysregulation. 
force microscopy to document membrane deformities, including flattening and ghost-like appearance in C. albicans yeast treated with thymol at the MIC or lower concentrations.

\section{CALCIUM BURSTS CORRELATE WITH ANTIFUNGAL EFFICACY}

Membrane-active antifungal agents such as amiodarone and carvacrol elicit dose-dependent bursts of cytosolic $\mathrm{Ca}^{2+}$ and downstream calcium-dependent stress response that appear to be directly in the pathway of fungal toxicity and cell death. We used the genetically encoded reporter, aequorin, reconstituted with its cofactor coelenterazine, to measure calcium fluxes in real time. Upon binding to $\mathrm{Ca}^{2+}$, the aequorin-coelenterazine complex emits light and luminescence intensity quantitatively correlates to free $\mathrm{Ca}^{2+}$ concentration. Addition of amiodarone or carvacrol resulted in characteristic spiking of free $\mathrm{Ca}^{2+}$ followed by a return to baseline. Removal of extracellular $\mathrm{Ca}^{2+}$ revealed that the primary source was from the medium, followed by a slower release from internal stores, likely to be the vacuole. The amplitude and duration of the $\mathrm{Ca}^{2+}$ burst correlated with drug toxicity. Thus, while similar bursts were observed with the structural isomers carvacrol and thymol, significantly lower bursts resulted from addition of the same concentration of eugenol. The remaining compounds tested (vanillin, guaiacol, $\gamma$-terpinene, and $p$-cymene) failed to elicit any luminescence change, consistent with their lack of toxicity (Rao et al., 2010).

In order to determine whether elevations of $\mathrm{Ca}^{2+}$ were a mere bystander effect or critical to mediating antifungal activity, we examined carvacrol toxicity in yeast mutants that were defective in calcium homeostasis. Thus, in vma $2 \Delta$ mutants that lack vacuolar $\mathrm{H}^{+}$pumping activity, $\mathrm{Ca}^{2+}$ cannot be effectively sequestered in vacuolar stores by $\mathrm{H}^{+} / \mathrm{Ca}^{2+}$ antiport mechanisms resulting in prolonged $\mathrm{Ca}^{2+}$ bursts in response to carvacrol. Sensitivity to carvacrol was concomitantly increased in $v m a 2 \Delta$ mutants, consistent with a mechanistic role for $\mathrm{Ca}^{2+}$ toxicity in the antifungal activity. A more thorough investigation of the link to $\mathrm{Ca}^{2+}$ bursts was made using the amphiphilic agent amiodarone. Low concentration of $\mathrm{Ca}^{2+}$ in the medium blocked cytosolic $\mathrm{Ca}^{2+}$ bursts and rescued growth inhibition by amiodarone (Muend and Rao, 2008). Chemical-genetic screening of the $S$. cerevisiae single-gene deletion library for hypersensitivity to amiodarone toxicity revealed novel pathways in which $\mathrm{Ca}^{2+}$ dysregulation was exacerbated, including pmrls mutants lacking the $\mathrm{Ca}^{2+}$ pumping ATPase of Golgi, and ergosterol biogenesis mutants (Gupta et al., 2003; Yadav et al., 2007). Conversely, in drug tolerant mutants defective in ceramide/sphingolipid biosynthesis, amiodarone-induced $\mathrm{Ca}^{2+}$ transients were suppressed (Table 1; Figure 2). Additional novel categories influencing drug toxicity included isoprenoid biosynthesis, fatty acid, and lipid transport, chromatin remodeling and vesicular trafficking (Gupta et al., 2003).

Other lines of evidence pointed to a critical role for $\mathrm{Ca}^{2+}$ bursts: dose-dependent loss of metabolic activity in amiodarone, measured using the vital dye FUN-1, was largely abrogated by removal of extracellular $\mathrm{Ca}^{2+}$ using the chelating agent BAPTA. We found antifungal efficacy to be dependent upon metabolic status, with stationary phase cells exhibiting low levels of $\mathrm{Ca}^{2+}$ influx and being more resistant to the drug. Addition of glucose was necessary for recovery of $\mathrm{Ca}^{2+}$ influx and regaining sensitivity to the
Table 1 | Genome-wide screen of $\boldsymbol{S}$. cerevisae collection of haploid deletion mutants for amiodarone-tolerant strains.

\begin{tabular}{|c|c|}
\hline Functional categories & Mutants \\
\hline $\begin{array}{l}\text { Sphingolipid } \\
\text { metabolism }\end{array}$ & $\begin{array}{l}\text { ipt1, yor170w (overlapping Icb4), Icb4, sur1, } \\
\text { csg2, ynl105w (overlapping inp52) }\end{array}$ \\
\hline $\begin{array}{l}\text { Nonsense-mediated } \\
\text { mRNA decay pathway }\end{array}$ & nmd2, upf3, nam7 \\
\hline $\begin{array}{l}\text { Transcriptional } \\
\text { regulation }\end{array}$ & sip3, hap3 \\
\hline $\begin{array}{l}\text { Programmed cell death } \\
\text { pathway }\end{array}$ & ysp1, ysp2 \\
\hline Miscellaneous & $\begin{array}{l}\text { sac6, ccs1, irc21, ymr102c, yor309c, orm2, } \\
\text { mfa1, yor } 364 w, y / r 407 w\end{array}$ \\
\hline
\end{tabular}

Methods were as described by Gupta et al. (2003) in the screen for hypersensitive strains, except that $18 \mu \mathrm{M}$ amiodarone was used. Wild type control failed to grow at this dose. Surviving strains are listed below. Note the enrichment of genes in lipid metabolism pathways, consistent with membrane effects of this drug.

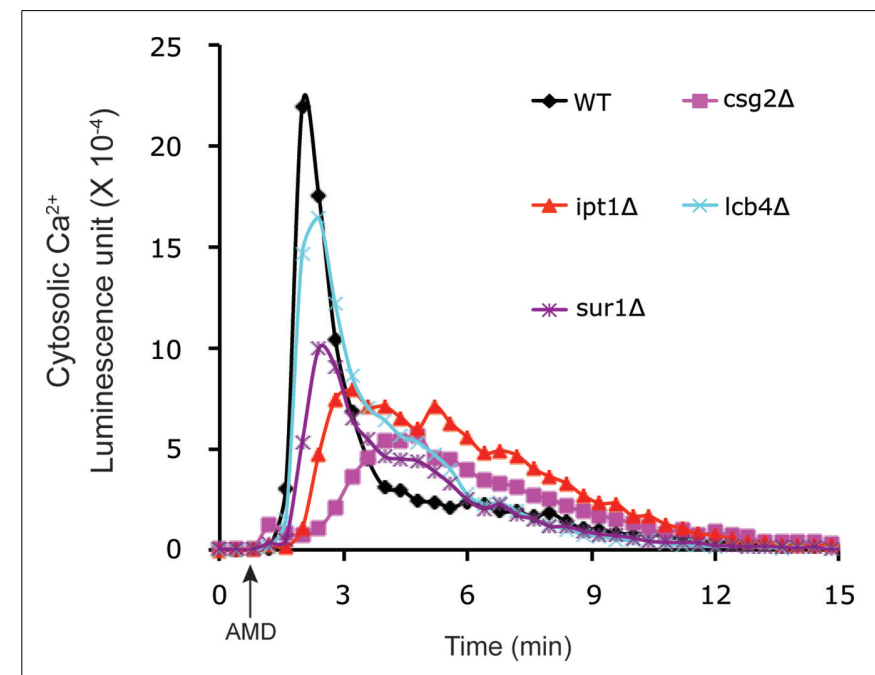

FIGURE 2 | Membrane-active antifungal drugs elicit $\mathbf{C a}^{2+}$ bursts.

Amiodarone (AMD, added at the arrow) elicits cytosolic $\mathrm{Ca}^{2+}$ transients in $S$. cerevisiae, detected by luminescence of aequorin-coelenterazine complex. Relative to the wild type control, four deletion mutants in the sphingolipid pathway, selected for amiodarone tolerance (Table 1), showed reduced or delayed $\mathrm{Ca}^{2+}$ bursts, consistent with the important role of ionic transients in mediating antifungal drug toxicity.

drug; this was prevented by treatment with cycloheximide, suggesting that new protein synthesis was required for replenishment of $\mathrm{Ca}^{2+}$ influx channels (Muend and Rao, 2008).

These observations illustrate the dual role of $\mathrm{Ca}^{2+}$ in the response to cell stress. On the one hand, $\mathrm{Ca}^{2+}$ influx occurs in response to a variety of environmental insults (osmotic stress, ER stress, cell wall damage, or prolonged exposure to mating factor). Elevation of baseline $\mathrm{Ca}^{2+}$ is sensed by effector proteins such as calmodulin and the $\mathrm{Ca}^{2+}$ /calmodulin dependent phosphatase calcineurin, that trigger a cellular response which includes cell wall 
synthesis, cell cycle arrest, and upregulation of ion transporters. However $\mathrm{Ca}^{2+}$ is a double-edged sword such that excessive elevation of $\mathrm{Ca}^{2+}$ is unequivocally toxic, triggering mitochondrial fragmentation, reactive oxygen species (ROS) generation, and cell death, in a way that can be exploited by antifungal agents.

\section{pH DYSREGULATION ELICITED BY CARVACROL AND MEMBRANE-ACTIVE COMPOUNDS}

Carvacrol hypersensitivity of vma2 $\Delta$ mutants, defective in $\mathrm{H}^{+}$ homeostasis mechanisms, prompted us to examine the effect of essential oil components on cellular $\mathrm{pH}$. We used compartmentspecific fluorescent probes to monitor $\mathrm{pH}$ changes in situ. Acetoxymethyl ester of the $\mathrm{pH}$-sensitive fluorescent compound, BCECF, is readily taken up by yeast cells, and becomes trapped by the action of esterases in the vacuole where it reports on changes in $\mathrm{pH}$. pHluorin is a genetically encoded $\mathrm{pH}$-sensitive version of GFP that can be used to monitor cytoplasmic $\mathrm{pH}$. We found that carvacrol elicited an immediate dose-dependent alkalinization of vacuolar $\mathrm{pH}$, accompanied by acidification of the cytosol indicating leakage of protons from the vacuole. A second, concentration dependent phase in these $\mathrm{pH}$ dependent changes was more precipitous, and occurred well downstream of the $\mathrm{Ca}^{2+}$ burst. Similar $\mathrm{pH}$ changes were observed with amiodarone: toxic levels of the drug elicited up to $0.4 \mathrm{pH}$ units of acidification in the cytoplasm that correlated with loss of viability. Interestingly, drug toxicity was significantly greater in actively metabolizing cells that had generated robust $\mathrm{pH}$ gradients across the plasma membrane. Amiodarone reversed these gradients, resulting in alkalinization of the medium and acidification of the cytoplasm. Stationary phase cells or cells starved of glucose failed to generate $\mathrm{pH}$ gradients and exhibited drug resistance (Muend and Rao, 2008).

These observations lead us to propose that membrane hyperpolarization triggers the opening of ion channels that bring in excess calcium leading to subsequent depolarization and $\mathrm{Ca}^{2+}$ stress. Indeed, early studies have demonstrated that $\mathrm{Ca}^{2+}$ influx is driven by $\Delta \Psi$. Glucose addition to depolarized cells triggered membrane polarization, $\mathrm{Ca}^{2+}$ influx and transient cytoplasmic acidification. Although the molecular identity of these $\mathrm{Ca}^{2+}$ influx channels is not known, there is precedence for hyperpolarization-gated and low $\mathrm{pH}$ dependent cation channels in plant cells (Hedrich et al., 1995; Bruggemann et al., 1999). Unlike conventional drugs, membrane-active compounds do not target specific proteins and may be less likely to be circumvented by development of fungal resistance. A genome-wide screen of amiodarone toxicity in the $S$. cerevisiae single-gene knockout collection revealed only a handful of yeast mutants that shifted MIC to higher drug concentrations by a modest amount (Table 1; Figure 2). It is worth noting that many of these fell in pathways that altered membrane lipid composition.

\section{TRANSCRIPTIONAL RESPONSES TO CARVACROL}

To gain further insight into cellular mechanisms involved in the antifungal activity of plant essential oils, we evaluated changes in mRNA transcripts in response to carvacrol concentrations that caused half-maximal $(0.005 \%)$ and maximal inhibition $(0.01 \%)$ of growth rates in $S$. cerevisiae. Transcripts were monitored in exponentially growing cells within $15 \mathrm{~min}$ of exposure to carvacrol, immediately following the bulk of the ionic fluxes that were observed. Among the most prominent categories significantly upregulated relative to control, were transcripts for alternative metabolic and energy handling pathways (glycogen and trehalose biosynthesis, polyamine degradation, and fatty acid transport and oxidation). As expected, many stress response and signaling pathways were induced, such as sporulation, oxygen and free radical detoxification, heat shock proteins and chaperones, autophagy, and vacuolar degradation mechanisms. Members of the $\mathrm{ABC}$ transporter family involved in drug efflux were also induced (SNQ2, YOR1, PDR5, PDR10, and PDR15). Fewer genes were downregulated and they were predominantly in the pathways of nucleic acid metabolism, showing that carvacrol rapidly shut down pathways of gene transcription, RNA processing, and modification, consistent with cessation of growth. The isomeric phenol thymol elicited a similar response, as reported by Bi et al. (2010) although there were some differences such as the repression of thymine biosynthesis and sulfur metabolism. Although these differences could be due to unique responses to structural isomers, we suggest that they are likely due to differences arising from late ( $90 \mathrm{~min}$ in the case of thymol) versus early ( $15 \mathrm{~min}$, carvacrol) transcriptional response.

Overall, the transcriptional response overlapped with $\mathrm{Ca}^{2+}$ stress and amiodarone toxicity, independently confirming the mechanistic importance of ionic fluxes in mediating the antifungal activity of carvacrol. Notably, the transcriptional profile differed from those of conventional antifungal drugs belonging to the classes of polyenes, azoles, allylamines, and morpholines. We also noted that the response to carvacrol resembled inhibition of the TOR pathway that controls growth in response to nutrient availability, although the specific link between the two pathways is not clear. Phenotype profiling of drug sensitivity/tolerance revealed cellular pathways upstream of transcriptional response, and did not show significant coincidence of identified genes. Together, these two genome-wide approaches provided complementary and non-overlapping mechanistic insight on genes and pathways involved in fungal death mediated by cationic amphiphiles.

\section{EXTENDING FROM MODEL TO PATHOGENIC FUNGI}

Studies in pathogenic yeast are hampered by inherent technical challenges, limited genetic resources and unavailability of molecular tools that delay advances in knowledge relating to pathogenesis. Therefore, we expanded our repertoire of $\mathrm{Ca}^{2+}, \mathrm{pH}$, and membrane potential reporters at the plasma membrane, mitochondria, cytosol, and vacuole to pathogenic Candida species, including C. albicans and C. glabrata. Genetically encoded reporters aequorin $\left(\mathrm{Ca}^{2+}\right)$ and pHluorin $\left(\mathrm{H}^{+}\right)$were codon optimized, tagged with compartment-specific localization signals (e.g., to mitochondria), expressed and validated in S. cerevisiae and Candida species as exemplified in Figures 3A-C. Similarly, we have optimized and validated uptake of compartment-specific reporter dyes (e.g., Figure 3D). These tools should be useful for future studies on the role of ion transients in mediating virulence or antifungal activity in both model and pathogenic yeasts.

\section{FUTURE PERSPECTIVES FOR ANTIFUNGAL THERAPY}

Azole compounds that target ergosterol biogenesis are among the most widely used antifungal drugs. Recent studies have shown 


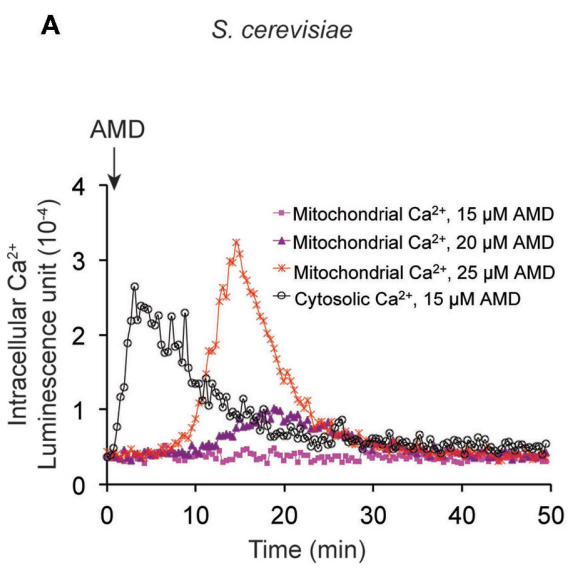

C

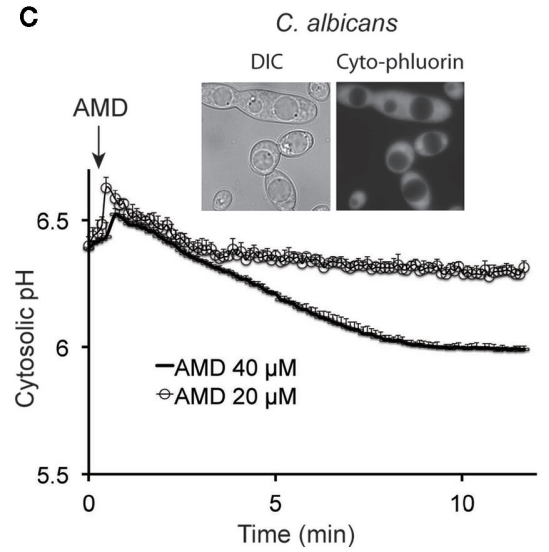

FIGURE 3 | Generation and validation of novel tools to monitor compartment-specific changes in $\mathrm{Ca}^{2+}$ and $\mathbf{p H}$. (A) Aequorin was targeted to cytoplasm or mitochondria in S. cerevisiae, by the addition of N-terminal signal sequence for the latter. Dose-dependent elevations of mitochondrial $\mathrm{Ca}^{2+}$ are seen to follow cytoplasmic $\mathrm{Ca}^{2+}$ fluxes upon amiodarone (AMD) addition (described previously, Gupta et al., 2003). (B) $\mathrm{pH}$-sensitive version of GFP, pHluorin, was targeted to mitochondria where it colocalized with MitoTracker Red as seen in the inset. Addition of amiodarone acidified mitochondrial matrix, relative to control,

that ergosterol depletion inhibits the activity of the V-type $\mathrm{H}^{+}$ATPase, without altering trafficking or assembly of the ATPase complex (Zhang et al., 2010). An important consequence of this inhibition is that azole drugs alkalinize the vacuole and exacerbate $\mathrm{Ca}^{2+}$ transients elicited by amiodarone, consistent with synergistic effects of these drugs (Gamarra et al., 2010; Zhang et al., 2010). Given that the V-ATPase is essential for virulence, these observations exemplify the importance of ionic gradients in pathophysiology of fungi (Zhang et al., 2010). Future studies could examine potential interactions between active components

\section{REFERENCES}

Antunes-Madeira, M. C., Videira, R. A., Kluppel, M. L., and Madeira, V. V. (1995). Amiodarone effects on membrane organization evaluated by fluorescence polarization. Int. J. Cardiol. 48, 211-218.
Ashley, E. S. D., Lewis, R., Lewis, J. S., Martin, C., and Andes, D. (2006). Pharmacology of systemic antifungal agents. Clin. Infect. Dis. 43, S28S39.

Bagshaw, R. J. (1983). Hypotension may have profound effect

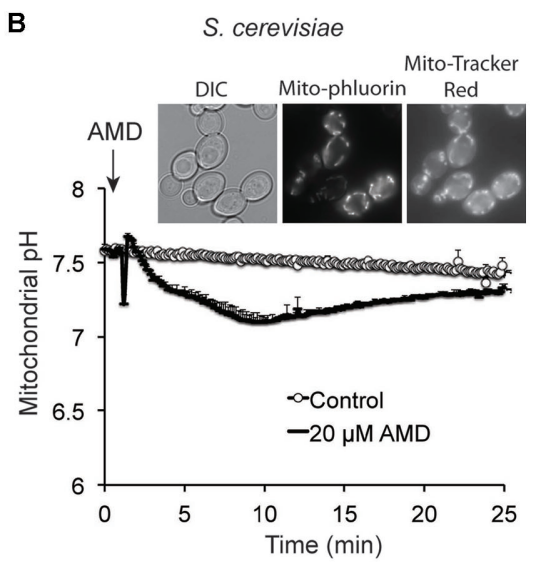

D

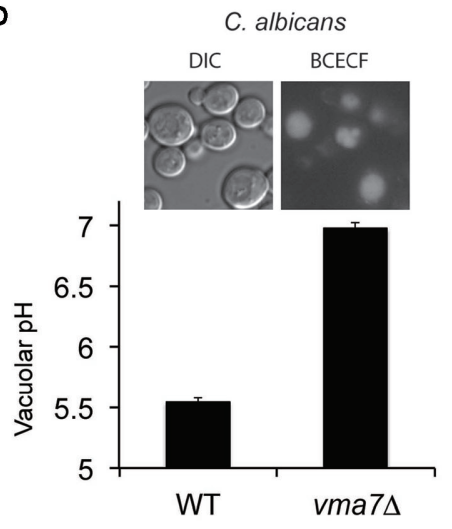

followed by slow recovery. (C) Cytosolic $\mathrm{pH}$ was monitored by expressing pHluorin in C. albicans, as seen in the inset. Addition of amiodarone elicits dose-dependent acidification as was previously reported in S. cerevisiae. (D) C. albicans vacuolar $\mathrm{pH}$ is monitored using the acetoxymethyl precursor of the $\mathrm{pH}$-sensitive fluorescent reporter BCECF, which can stably accumulate in vacuoles (inset) through the action of esterases. The reporter was validated by showing alkalinization of vacuolar $\mathrm{pH}$ in $\mathrm{vma}^{-/-}$mutants, lacking a functional V-ATPase. DIC, differential interference contrast.

of plant essential oils and azoles or other classes of antifungals. In addition, carvacrol and other terpenoid phenols could be tested for chemosensitization in conjunction with calcineurin inhibitors (cyclosporine A, FK506) that enhance $\mathrm{Ca}^{2+}$ dysregulation, and rapamycin analogs that block TOR signaling, against pathogenic fungi.

\section{ACKNOWLEDGMENTS}

This work was supported by a grant from the National Institutes of Health R01AI065983.

on the liver. Anesthesiology 59, 168-169.

Ben Arfa, A., Combes, S., PreziosiBelloy, L., Gontard, N., and Chalier, P. (2006). Antimicrobial activity of carvacrol related to its chemical structure.
Lett. Appl. Microbiol. 43, 149-154.

Bi, X., Guo, N., Jin, J., Liu, J., Feng, H., Shi, J., Xiang, H., Wu, X., Dong, J., Hu, H., Yan, S., Yu, C., Wang, X., Deng, X., and Yu, L. (2010). The global gene expression profile of the 
model fungus Saccharomyces cerevisiae induced by thymol. J. Appl. Microbiol. 108, 712-722.

Blankenship, J. R., and Heitman, J. (2005). Calcineurin is required for Candida albicans to survive calcium stress in serum. Infect. Immun. 73, 5767-5774.

Braga, P. C., and Ricci, D. (2011). Thymol-induced alterations in Candida albicans imaged by atomic force microscopy. Methods Mol. Biol. 736, 401-410.

Brett, C. L., Tukaye, D. N., Mukherjee, S., and Rao, R. (2005). The yeast endosomal $\mathrm{Na}+\mathrm{K}+/ \mathrm{H}+$ exchanger $\mathrm{Nhx} 1$ regulates cellular $\mathrm{pH}$ to control vesicle trafficking. Mol. Biol. Cell 16, 1396-1405.

Bruggemann, L., Dietrich, P., Becker, D., Dreyer, I., Palme, K., and Hedrich, R. (1999). Channel-mediated highaffinity $\mathrm{K}+$ uptake into guard cells from Arabidopsis. Proc. Natl. Acad. Sci. U.S.A. 96, 3298-3302.

Dalleau, S., Cateau, E., Berges, T., Berjeaud, J. M., and Imbert, C. (2008). In vitro activity of terpenes against Candida biofilms. Int. J. Antimicrob. Agents 31, 572-576.

Davis, D. A. (2009). How human pathogenic fungi sense and adapt to $\mathrm{pH}$ : the link to virulence. Curr. Opin. Microbiol. 12, 365-370.

De Martino, L., De Feo, V., Fratianni, F., and Nazzaro, F. (2009). Chemistry, antioxidant, antibacterial and antifungal activities of volatile oils and their components. Nat. Prod. Commun. 4, 1741-1750.

Francois, I. E., Aerts, A. M., Cammue, B. P., and Thevissen, K. (2005). Currently used antimycotics: spectrum, mode of action and resistance occurrence. Curr. Drug Targets 6, 895-907.

Gamarra, S., Rocha, E. M., Zhang, Y. Q., Park, S., Rao, R., and Perlin, D. S. (2010). Mechanism of the synergistic effect of amiodarone and fluconazole in Candida albicans. Antimicrob. Agents Chemother. 54, 1753-1761.

Gudlaugsson, O., Gillespie, S., Lee, K., Vande Berg, J., Hu, J., Messer,
S., Herwaldt, L., Pfaller, M., and Diekema, D. (2003). Attributable mortality of nosocomial candidemia, revisited. Clin. Infect. Dis. 37, 1172-1177.

Gupta, S. S., Ton, V. K., Beaudry, V., Rulli, S., Cunningham, K., and Rao, R. (2003). Antifungal activity of amiodarone is mediated by disruption of calcium homeostasis. J. Biol. Chem. 278, 28831-28839.

Hedrich, R., Moran, O., Conti, F., Busch, H., Becker, D., Gambale, F., Dreyer, I., Kuch, A., Neuwinger, K., and Palme, K. (1995). Inward rectifier potassium channels in plants differ from their animal counterparts in response to voltage and channel modulators. Eur. Biophys. J. 24, 107-115.

Kraus, P. R., and Heitman, J. (2003). Coping with stress: calmodulin and calcineurin in model and pathogenic fungi. Biochem. Biophys. Res. Commun. 311, 1151-1157.

Lew, R. R. (2011). How does a hypha grow? The biophysics of pressurized growth in fungi. Nat. Rev. Microbiol. 9, 509-518.

Maresova, L., Muend, S., Zhang, Y. Q., Sychrova, H., and Rao, R. (2009). Membrane hyperpolarization drives cation influx and fungicidal activity of amiodarone. J. Biol. Chem. 284, 2795-2802.

Mastelic, J., Jerkovic, I., Blazevic, I., Poljak-Blazi, M., Borovic, S., IvancicBace, I., Smrecki, V., Zarkovic, N., Brcic-Kostic, K., Vikic-Topic, D., and Muller, N. (2008). Comparative study on the antioxidant and biological activities of carvacrol, thymol, and eugenol derivatives. J. Agric. Food Chem. 56, 3989-3996.

Morace, G., and Borghi, E. (2010). Fungal infections in ICU patients: epidemiology and the role of diagnostics. Minerva Anestesiol. 76, 950-956.

Muend, S., and Rao, R. (2008). Fungicidal activity of amiodarone is tightly coupled to calcium influx. FEMS Yeast Res. 8, 425-431.

Pappas, P. G., Kauffman, C. A., Andes, D., Benjamin, D. K. Jr., Calandra,
T. F., Edwards, J. E. Jr., Filler, S. G., Fisher, J. F., Kullberg, B. J., OstroskyZeichner, L., Reboli, A. C., Rex, J. H., Walsh, T. J., and Sobel, J. D. (2009). Clinical practice guidelines for the management of candidiasis: 2009 update by the Infectious Diseases Society of America. Clin. Infect. Dis. 48, 503-535.

Pinto, E., Vale-Silva, L., Cavaleiro, C., and Salgueiro, L. (2009). Antifungal activity of the clove essential oil from Syzygium aromaticum on Candida, Aspergillus and dermatophyte species. J. Med. Microbiol. 58, 1454-1462.

Pozzatti, P., Scheid, L. A., Spader, T. B., Atayde, M. L., Santurio, J. M., and Alves, S. H. (2008). In vitro activity of essential oils extracted from plants used as spices against fluconazole-resistant and fluconazole-susceptible Candida spp. Can. J. Microbiol. 54, 950-956.

Rao, A., Zhang, Y., Muend, S., and Rao, R. (2010). Mechanism of antifungal activity of terpenoid phenols resembles calcium stress and inhibition of the TOR pathway. Antimicrob. Agents Chemother. 54, 5062-5069.

Soong, T. W., Yong, T. F., Ramanan, N., and Wang, Y. (2000). The Candida albicans antiporter gene $\mathrm{CNH} 1$ has a role in $\mathrm{Na}+$ and $\mathrm{H}+$ transport, salt tolerance, and morphogenesis. Microbiology 146, 1035-1044.

Tampieri, M. P., Galuppi, R., Macchioni, F., Carelle, M. S., Falcioni, L., Cioni, P. L., and Morelli, I. (2005). The inhibition of Candida albicans by selected essential oils and their major components. Mycopathologia 159, 339-345.

Ultee, A., Bennik, M. H., and Moezelaar, R. (2002). The phenolic hydroxyl group of carvacrol is essential for action against the food-borne pathogen Bacillus cereus. Appl. Environ. Microbiol. 68, 1561-1568.

Voda, K., Boh, B., and Vrtacnik, M. (2004). A quantitative structureantifungal activity relationship study of oxygenated aromatic essential oil compounds using data structuring and PLS regression analysis. J. Mol. Model. 10, 76-84.

Wang, H., Liang, Y., Zhang, B., Zheng, W., Xing, L., and Li, M. (2011). Alkaline stress triggers an immediate calcium fluctuation in Candida albicans mediated by Rim101p and Crzlp transcription factors. FEMS Yeast Res. 11, 430-439.

Xu, J., Zhou, F., Ji, B. P., Pei, R. S., and $\mathrm{Xu}, \mathrm{N}$. (2008). The antibacterial mechanism of carvacrol and thymol against Escherichia coli. Lett. Appl. Microbiol. 47, 174-179.

Yadav, J., Muend, S., Zhang, Y., and Rao, R. (2007). A phenomics approach in yeast links proton and calcium pump function in the golgi. Mol. Biol. Cell 18, 1480-1489.

Zhang, Y. Q., Gamarra, S., GarciaEffron, G., Park, S., Perlin, D. S., and Rao, R. (2010). Requirement for ergosterol in V-ATPase function underlies antifungal activity of azole drugs. PLoS Pathog. 6, e1000939. doi:10.1371/journal.ppat.1000939

Conflict of Interest Statement: The authors declare that the research was conducted in the absence of any commercial or financial relationships that could be construed as a potential conflict of interest.

Received: 15 February 2012; paper pending published: 23 February 2012; accepted: 20 March 2012; published online: 05 April 2012.

Citation: Zhang Y, Muend $S$ and Rao $R$ (2012) Dysregulation of ion homeostasis by antifungal agents. Front. Microbio. 3:133. doi: 10.3389/fmicb.2012.00133

This article was submitted to Frontiers in Fungi and Their Interactions, a specialty of Frontiers in Microbiology. Copyright (c) 2012 Zhang, Muend and Rao. This is an open-access article distributed under the terms of the Creative Commons Attribution Non Commercial License, which permits noncommercial use, distribution, and reproduction in other forums, provided the original authors and source are credited. 